\title{
Heteronuclear ionizing collisions between laser-cooled metastable helium atoms
}

\author{
J. M. McNamara, R. J. W. Stas, W. Hogervorst, and W. Vassen \\ Department of Physics and Astronomy, Laser Centre Vrije Universiteit, \\ De Boelelaan 1081, $1081 \mathrm{HV}$ Amsterdam, The Netherlands
}

(Dated: October 18, 2018)

\begin{abstract}
We have investigated cold ionizing heteronuclear collisions in dilute mixtures of metastable $\left(2{ }^{3} S_{1}\right){ }^{3} \mathrm{He}$ and ${ }^{4} \mathrm{He}$ atoms, extending our previous work on the analogous homonuclear collisions [R. J. W. Stas et al., PRA 73, 032713 (2006)]. A simple theoretical model of such collisions enables us to calculate the heteronuclear ionization rate coefficient, for our quasi-unpolarized gas, in the absence of resonant light $(T=1.2 \mathrm{mK}): K_{34}^{(\mathrm{th})}=2.4 \times 10^{-10} \mathrm{~cm}^{3} / \mathrm{s}$. This calculation is supported by a measurement of $K_{34}$ using magneto-optically trapped mixtures containing about $1 \times 10^{8}$ atoms of each species, $K_{34}^{(\exp )}=2.5(8) \times 10^{-10} \mathrm{~cm}^{3} / \mathrm{s}$. Theory and experiment show good agreement.

PACS numbers: 32.80.Pj, 34.50.Fa, 34.50.Rk
\end{abstract}

\section{INTRODUCTION}

The importance of cold collisions with regard to the dynamics of dilute neutral atom clouds was realized soon after the advent of laser-cooled and trapped atomic samples [1]. Since then, numerous investigations, both experimental and theoretical, have been made into the collisional properties of many different homonuclear [2, 3, 4, 5, 6, 7, 8, 9, 10, 11, 12, 13] and (later) heteronuclear [14, 15, 16, 17, 18, 19] systems. Collisional studies are in themselves interesting, leading to an in-depth understanding of the various scattering mechanisms present at such low kinetic energies and methods by which we can have some measure of control over elastic and inelastic collisions.

As opposed to collisions between thermal atoms, collisions between cold atoms are sensitive to the longrange part of the interatomic interaction potential. The de Broglie wavelength may become comparable to the characteristic range of the interatomic potential, and (in the presence of a light field) the possibility of exciting a quasi-molecular state, and the subsequent decay of that state during a collision, becomes important. These effects lead to phenomena such as scattering resonances, interaction retardation, optically assisted collisions, photoassociation, optical shielding and the formation of groundstate molecules.

Optically assisted collisions lead to large losses in magneto-optical traps (MOTs) and are clearly dependant upon experimental parameters such as the intensity and detuning of the light frequencies present. A full theoretical treatment of these collisions is often hampered by the complexity of the molecular hyperfine structure [1], thus MOTs are usually empirically optimized for their intended application, and comparisons with theory or other experiments are difficult. Collisions in the absence of a resonant light field are, on the other hand, a theoretically more tractable problem and their associated loss rate coefficients are fundamental properties of a given system, allowing direct comparisons with theory and between experiments. Homonuclear and heteronuclear collisions, "in the dark", between isotopes of a single element are both mediated at long-range by the van-der-Waals interaction $\left(\propto 1 / \mathrm{R}^{6}\right)$ and any differences are due, in the main, to differing atomic structures and quantum statistical symmetries.

Due to the high internal energy $(19.8 \mathrm{eV})$ of metastable $\left(2{ }^{3} S_{1}\right)$ helium atoms $\left(\mathrm{He}^{*}\right)$, the spherical symmetry of this atomic state, and the inverted (hyper)fine structure of the atoms; ionizing collisions (Penning (PI) and associative $(\mathrm{AI}))$ :

$$
\begin{aligned}
& \mathrm{He}^{*}+\mathrm{He}^{*} \rightarrow \mathrm{He}+\mathrm{He}^{+}+e^{-}(\mathrm{PI}), \\
& \mathrm{He}^{*}+\mathrm{He}^{*} \rightarrow \mathrm{He}_{2}^{+}+e^{-} \quad(\mathrm{AI}),
\end{aligned}
$$

dominate losses in trapped samples of laser-cooled $\mathrm{He}^{*}$. This has provided a unique setting for the study of cold ionizing collisions in which the highly efficient, direct detection of collisional loss products using charged-particle detectors is possible. In the past, several experiments have made use of microchannel plate (MCP) detectors to measure ion production rates and investigate collisional losses in ${ }^{3} \mathrm{He}^{*}$ [20] and ${ }^{4} \mathrm{He}^{*}$ [20, 21, 22, 23]. Having realized the ability to trap large numbers $\left(>10^{8}\right)$ of both isotopes (either individually or simultaneously) [19], we have previously reported on the isotopic differences between binary homonuclear collisions of ${ }^{3} \mathrm{He}$ and ${ }^{4} \mathrm{He}[13]$ in the absence of resonant light, resolving inconsistencies in prior experimental and theoretical results.

In this article we describe what we believe to be the first study of heteronuclear binary collisions between metastable atoms. Adapting our transparent theoretical model [13] slightly, we first derive a value for $K_{34}$ (Sec. III), the heteronuclear ionization rate coefficient in the absence of a resonant light field. This is complemented by trap loss measurements performed on a two-isotope magneto-optical trap (TIMOT) of ${ }^{3} \mathrm{He}^{*}$ and ${ }^{4} \mathrm{He}^{*}$ [19], from which we also extract a value for $K_{34}$ (Sec. IV). In Section $\mathrm{V}$ we compare both results and briefly comment upon loss rates in the presence of the trapping light fields. 


\section{SINGLE CHANNEL MODEL OF THE HETERONUCLEAR IONIZING LOSS RATE}

The following model has been described in depth elsewhere in its successful application to the description of homonuclear ionizing collisions [13, 24], where it compares well with the more comprehensive close coupling theories developed by Venturi et al. [25, 26] and Leo et al. [27]. Here we extend its applicability to include the description of heteronuclear He* collisions for which no calculations have previously been made. In the interests of brevity we only describe the models salient points and its adaption to the heteronuclear case.

\section{A. Ionization rate coefficients}

At $\mathrm{mK}$ temperatures, collisional processes are dominated by only a few partial waves, $\ell$, and the ionization cross section may be written as a sum over the partial wave contributions:

$$
\sigma^{(\mathrm{ion})}=\sum_{\ell} \sigma_{\ell}^{(\mathrm{ion})}
$$

From a semi-classical viewpoint we may further treat the inelastic scattering as a two-stage process in which (at low energies) elastic scattering from the interaction potential $V(R)$ occurs at relatively large internuclear distance $\left(R \geq 100 a_{0}\right)$, whilst ionization occurs only at small internuclear distance $\left(R \approx 5 a_{0}\right)$ [28]. The assumption that the processes of elastic scattering and ionization are uncoupled allows us to factorize the probability of ionization occurring in a collision, and write the ionization cross section for collisions with total electronic spin $S$ as

$$
{ }^{(2 S+1)} \sigma^{(\text {ion })}=\frac{\pi}{k^{2}} \sum_{\ell}(2 \ell+1)^{(2 S+1)} P_{\ell}^{(\text {tun })(2 S+1)} P^{(\text {ion })}
$$

where $k$ is the wave vector of the relative motion of the two atoms, ${ }^{(2 S+1)} P_{\ell}^{\text {(tun) }}$ is the probability of the atoms reaching small internuclear distance (i.e., not elastically scattering) and ${ }^{(2 S+1)} P^{(\text {ion })}$ is the probability of ionization occurring at short internuclear distance. In the $\mathrm{He}^{*}$ system the quantum number $S$ is well conserved during ionization and the application of Wigner's spin conservation rule 29] tells us that ${ }^{5} P^{(\text {ion })}$ is very small 30], while Müller et al. 31] report ionization probabilities of 0.975 for other spin states; we thus set ${ }^{5} P^{(\text {ion })}=0$ and ${ }^{1} P^{(\text {ion })}={ }^{3} P^{(\text {ion })}=1$ in Eq. (2). Having constructed the ${ }^{1} \Sigma_{g}^{+},{ }^{3} \Sigma_{u}^{+}$and ${ }^{5} \Sigma_{g}^{+}$molecular potentials as described in [27] (to which we may add rotational barriers as required) we modify them to simulate the losses due to ionization (Fig. 11) 32]. By numerically solving the radial wave equation and finding the stationary states using these potentials, we may calculate the incident and transmitted probability currents, $J_{\text {in }}$ and $J_{\text {tr }}$ respectively, the ratio of which $\left(J_{\mathrm{tr}} / J_{\text {in }}\right)$ gives us ${ }^{(2 S+1)} P_{\ell}^{(\mathrm{tun})}$. The calcu-

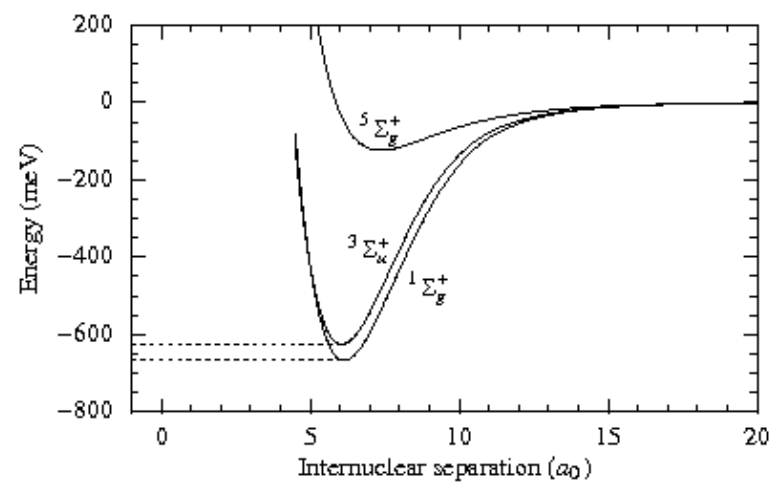

FIG. 1: He* potentials (labeled in Hund's case (a) notation) constructed as described in [27] (solid), together with the modifications made (dashed). Atoms reaching the region of small $R$ ionize; the corresponding relative particle propagates freely to $R=-\infty$ in our model, and ionization is accounted for by the loss of probability flux from the region of the potential well.

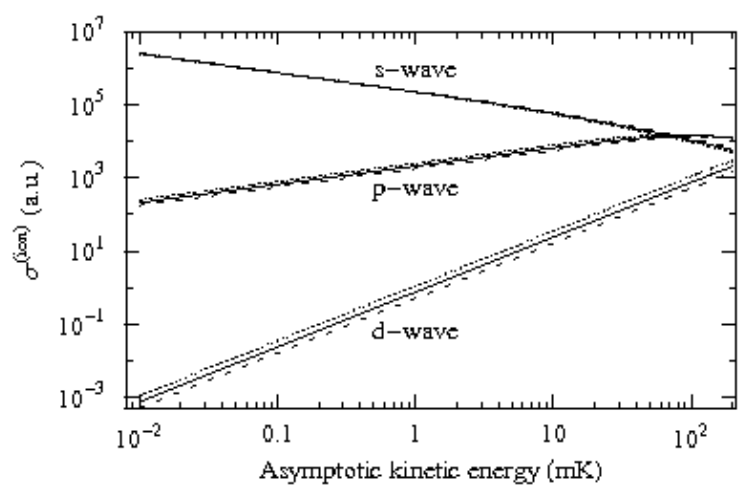

FIG. 2: Partial wave ionization cross sections $(S=0)$ for ${ }^{3} \mathrm{He}^{*}{ }_{-}^{4} \mathrm{He}^{*}$ (solid lines), ${ }^{3} \mathrm{He}^{*}{ }^{3} \mathrm{He}^{*}$ (dotted lines) and ${ }^{4} \mathrm{He}^{*}{ }_{-}^{4} \mathrm{He}^{*}$ (dashed lines). The familiar quantum threshold behavior $\left(\sigma_{\ell}^{\text {(ion) }} \propto k^{2 \ell-1}(k \rightarrow 0)\right)$ is displayed.

lated partial wave ionization cross sections are shown in Fig. 2,

The ionization rate coefficient $K$ (particle ${ }^{-1} \mathrm{~cm}^{3} / \mathrm{s}$ ) (determined in experiments) is temperature dependant, and may be written in terms of the ionization cross section $\sigma^{(\text {ion })}(E)[1,32,33]$ :

$$
K(T)=\int_{0}^{\infty} \sigma^{(\mathrm{ion})}(E) P_{T}^{(\mathrm{MB})}\left(v_{r}\right) v_{r} d v_{r},
$$

where $P_{T}^{(\mathrm{MB})}\left(v_{r}\right)$ is the Maxwell-Boltzmann distribution, for a given temperature, of the relative velocities in the atomic sample. Similarly, we may calculate the partial wave ionization rate coefficients ${ }^{(2 S+1)} \mathcal{K}_{\ell}(T)$ associated with a given molecular state (ignoring the radial contributions: $\left.\left|s_{1} i_{1}, s_{2}, S, F M_{F}, \ell m_{\ell}\right\rangle\right)$ [32].

Although the calculated values of ${ }^{(2 S+1)} \sigma_{\ell}^{\text {(ion) }}$ (Fig. 2), and ${ }^{(2 S+1)} \mathcal{K}_{\ell}(T)$, are very similar for all isotopic combinations, the description of the collisional process in terms 
TABLE I: Expansion coefficients $a_{S I}(F)=$ $\left\langle s_{1} i_{1}, s_{2}, S, F M_{F}, \ell m_{\ell} \mid s_{1} i_{1} f_{1}, s_{2} j_{2}, F M_{F}, \ell m_{\ell}\right\rangle$. The scattering states $\left|s_{1} i_{1} f_{1}, s_{2} j_{2}, F M_{F}, \ell m_{\ell}\right\rangle$ are indicated by their values of $F$, while the molecular states $\left|s_{1} i_{1}, s_{2}, S, F M_{F}, \ell m_{\ell}\right\rangle$ are given in Hund's case (a) notation, ${ }^{2 S+1} \Sigma_{\mathrm{g} / \mathrm{u}}^{+}$.

\begin{tabular}{lccc}
\hline \hline $\mathrm{F}$ & ${ }^{1} \Sigma_{\mathrm{g}}^{+}$ & ${ }^{3} \Sigma_{\mathrm{u}}^{+}$ & ${ }^{5} \Sigma_{\mathrm{g}}^{+}$ \\
\hline $1 / 2$ & $\sqrt{2 / 3}$ & $-\sqrt{1 / 3}$ & \\
$3 / 2$ & & $\sqrt{5 / 6}$ & $-\sqrt{1 / 6}$ \\
$5 / 2$ & & & 1 \\
\hline \hline
\end{tabular}

of partial waves for each combination is very different due to the differing quantum statistics involved. In the case of homonuclear collisions the symmetrization postulate limits the number of physical scattering states describing a colliding pair. However, in the heteronuclear case there is no symmetry requirement and all partial wave contributions must be taken into account.

Because the energy gained during a collision is so large, the evolution of an atomic state $\left(\left|s_{1} i_{1} f_{1}, s_{2} j_{2}, F M_{F}, \ell m_{\ell}\right\rangle\right)$ in the region where the atomic hyperfine interaction is of the same order of magnitude as the molecular interaction may be described to a good approximation as being diabatic. Thus, to determine the ionization rate associated with a given scattering state $K(F)$, we simply expand the atomic states onto the eigenstates of the short-range molecular Hamiltonian,

$$
\begin{aligned}
\left|s_{1} i_{1} f_{1}, s_{2} j_{2}, F M_{F}, \ell m_{\ell}\right\rangle= & \sum_{S, I} a_{S I}(F) \times \\
& \left|s_{1} i_{1}, s_{2}, S, F M_{F}, \ell m_{\ell}\right\rangle,
\end{aligned}
$$

determine the fraction of ionizing states $(S=0,1)$ in this expansion (see Table I), and sum over the contributing partial waves:

$$
K(F)=\sum_{\ell} \sum_{S, I}\left|a_{S I}(F)\right|^{2} \times{ }^{(2 S+1)} \mathcal{K}_{\ell}
$$

Using the partial wave ionization rates obtained we may then derive the total ionization rate coefficient for an unpolarized sample, i.e., the rate coefficient for which the magnetic substates of the atoms in our sample are evenly populated:

$$
K^{(\text {unpol })}=\frac{1}{\left(2 f_{1}+1\right)} \frac{1}{\left(2 f_{2}+1\right)} \sum_{F} \sum_{M_{F}} K(F),
$$

which in the heteronuclear case gives:

$$
K_{34}^{(\text {unpol })} \approx \frac{1}{12}\left[\frac{4}{3}\left({ }^{1} \mathcal{K}_{0}+{ }^{1} \mathcal{K}_{1}\right)+4\left({ }^{3} \mathcal{K}_{0}+{ }^{3} \mathcal{K}_{1}\right)\right]
$$

The energy dependant unpolarized ionization rate coef-

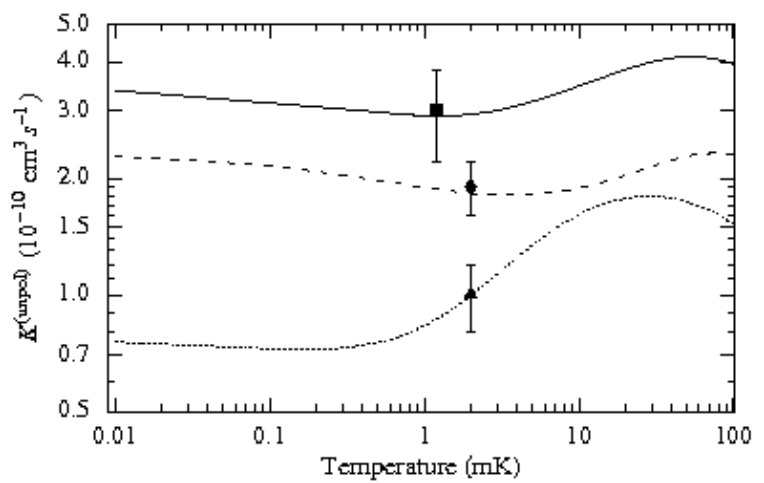

FIG. 3: Theoretical unpolarized loss rate coefficient curves: ${ }^{3} \mathrm{He}-{ }^{3} \mathrm{He}$ (dashed), ${ }^{4} \mathrm{He}-{ }^{4} \mathrm{He}$ (dotted) and, ${ }^{3} \mathrm{He}-{ }^{4} \mathrm{He}$ (solid), together with our experimental data points and their error bars: ${ }^{3} \mathrm{He}-{ }^{3} \mathrm{He}$ (diamond), ${ }^{4} \mathrm{He}-{ }^{4} \mathrm{He}$ (triangle) and ${ }^{3} \mathrm{He}-{ }^{4} \mathrm{He}$ (square). For the purposes of this figure, the experimental points have been corrected for the inhomogeneous distribution over the magnetic substates found in our MOT's (see Sec. IIB).

ficients are shown in Fig. 3 (including those rates previously calculated [13] for ${ }^{3} \mathrm{He}^{*}$ and $\left.{ }^{4} \mathrm{He}^{*}\right)$ and for a temperature of $T=1 \mathrm{mK}$ we obtain

$$
K_{34}^{(\text {unpol })}=2.9 \times 10^{-10} \mathrm{~cm}^{3} / \mathrm{s} .
$$

\section{B. Ionization rate coefficient of trapped samples}

Optical pumping processes in a MOT cause the distribution over magnetic substates $P_{m}$ (where $m$ is the azimuthal quantum number) of the trapped atoms to differ from the uniform (unpolarized) distribution assumed above. This is important as the contribution of each collision channel to the ionizing losses depends upon $P_{m}$, and can be accounted for in our theoretical model by using the density operator 34 ]

$$
\rho(\boldsymbol{r})=\sum_{m} \sum_{n \leq m} P_{m}(\boldsymbol{r}) P_{n}(\boldsymbol{r})|m, n\rangle\langle m, n|
$$

to describe a statistical mixture of magnetic substate pairs $|m, n\rangle$, where $m$ and $n$ are the azimuthal quantum numbers of the colliding atoms. The ionization rate coefficient of the mixture can then be written as

$$
K_{34}=\frac{1}{N_{3} N_{4}} \iiint\left(\sum_{\ell}\left({ }^{1} b^{1} \mathcal{K}_{\ell}+{ }^{3} b^{3} \mathcal{K}_{\ell}\right)\right) n_{3}(\boldsymbol{r}) n_{4}(\boldsymbol{r}) \mathrm{d}^{3} r
$$

where $N_{3}$ and $N_{4}$ are the number of trapped atoms in each component of the mixture, the coefficients ${ }^{(2 S+1)} b$ are the sums of the expectation values of the density operator for all ionizing molecular states with total spin $S$, and, $n_{3}(\boldsymbol{r})$ and $n_{4}(\boldsymbol{r})$ are the density distributions of each component in the sample. Explicit expressions for the coefficients ${ }^{(2 S+1)} b$ are given in Table II One may 
TABLE II: ${ }^{3} \mathrm{He}^{*}{ }^{4} \mathrm{He}^{*}{ }^{(2 S+1)} b$ coefficients from Eq. (10). The coefficients are the expectation values of the density operator, Eq. (9), for all ionizing molecular states of given $S$ and parity.

\begin{tabular}{cc}
\hline \hline${ }^{(2 S+1)} b$ & ${ }^{3} \mathrm{He}^{*}{ }^{4} \mathrm{He}^{*}$ \\
\hline${ }^{1} b$ & $(1 / 3)\left(P_{-3 / 2} P_{1}+P_{3 / 2} P_{-1}\right)+(2 / 9)\left(P_{-1 / 2} P_{0}+P_{1 / 2} P_{0}\right)+(1 / 9)\left(P_{-1 / 2} P_{1}+P_{1 / 2} P_{-1}\right)$ \\
${ }^{3} b$ & $(1 / 2)\left(P_{-3 / 2} P_{1}+P_{-3 / 2} P_{0}+P_{-1 / 2} P_{1}+P_{1 / 2} P_{-1}+P_{3 / 2} P_{0}+P_{3 / 2} P_{-1}\right)$ \\
& $+(1 / 3)\left(P_{-1 / 2} P_{-1}+P_{1 / 2} P_{1}\right)+(1 / 6)\left(P_{-1 / 2} P_{0}+P_{1 / 2} P_{0}\right)$ \\
\hline \hline
\end{tabular}

easily check that we recover Eq. (7) from Eq. (10) by substituting the values $P_{-3 / 2}=P_{-1 / 2}=P_{1 / 2}=P_{3 / 2}=$ $\frac{1}{4}$ and $P_{-1}=P_{0}=P_{1}=\frac{1}{3}$ into the expressions for the coefficients ${ }^{(2 S+1)} b$ (Table II) and evaluating Eq. (10).

In order to later make a comparison between theory and experiment, we determine the distribution $P_{m}(\boldsymbol{r})$ by obtaining the steady-state solution of a rate equation model describing the optical pumping in our MOT [23]. At a temperature of $T=1.2 \mathrm{mK}$ the resulting value of the theoretical ionization rate coefficient is

$$
K_{34}^{(\mathrm{th})}=2.4 \times 10^{-10} \mathrm{~cm}^{3} / \mathrm{s}
$$

\section{EXPERIMENTAL SETUP}

We investigate cold ionizing collisions in a setup (see Fig. 4) capable of trapping large numbers $\left(\gtrsim 10^{8}\right)$ of both ${ }^{3} \mathrm{He}^{*}$ and ${ }^{4} \mathrm{He}^{*}$ atoms simultaneously in a TIMOT [13]. A collimated and Zeeman slowed $\mathrm{He}^{*}$ beam is used to load our TIMOT which is housed inside a stainless steel, ultra-high vacuum chamber. The beam is produced by a liquid nitrogen $\left(\mathrm{LN}_{2}\right)$ cooled dc discharge source supplied with an isotopically enriched $(\approx 50 / 50)$ gaseous mixture of ${ }^{3} \mathrm{He}$ and ${ }^{4} \mathrm{He}$ held in a helium tight reservoir. During operation the reservoir is connected such that all helium not entering the collimation section (the vast majority of it) is pumped back into the reservoir and recycled, conserving our supply of the relatively expensive ${ }^{3} \mathrm{He}$ gas. Two $\mathrm{LN}_{2}$ cooled molecular sieves, ensuring a pure supply of helium to the source, are also contained within this reservoir; the first of these is a type $13 \mathrm{X}$ molecular sieve, whilst the second is type $4 \mathrm{~A}$ (both are sodium zeolites having pore sizes of $10 \AA$ and $4 \AA$ respectively). We then make use of the curved wavefront technique to collimate our atomic beam in two dimensions [35] before it enters the Zeeman slower. Due to its lighter mass ${ }^{3} \mathrm{He}$ atoms emerge from the source with a greater mean velocity than ${ }^{4} \mathrm{He}$ atoms and in order to achieve a large flux of both ${ }^{3} \mathrm{He}^{*}$ and ${ }^{4} \mathrm{He}^{*}$ atoms we have increased the capture velocity of our Zeeman slower [19, 24]. Our ultra-high vacuum chamber maintains an operational pressure of $7 \times 10^{-10} \mathrm{mbar}$ (with a partial presure of $6.5 \times 10^{-10} \mathrm{mbar}$, ground state He atoms from the atomic beam are the major contribution to this) and is based upon the design of our next-generation BEC chamber 36]. Two coils in an anti-Helmholtz configuration produce the quadrupole

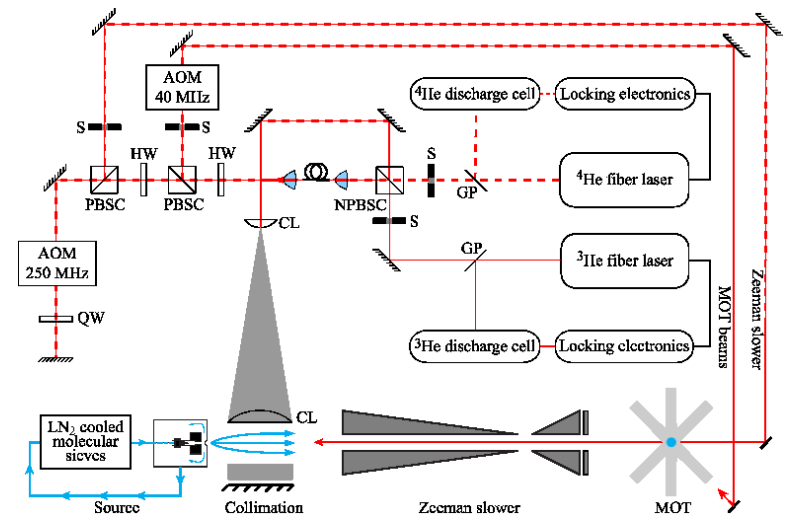

FIG. 4: A schematic overview of the TIMOT experimental apparatus used in these experiments. Component labels are: AOM, acousto-optic modulator; QW, quater-wave plate; S, shutter; PBSC, polarizing beam splitter cube; HW, half-wave plate; CL, cylindrical lens; and NPBSC, non-polarizing beamsplitter cube. For clarity, all spherical lenses and excess mirrors have been omitted.

magnetic field $(d B / d z=0.35 \mathrm{~T} / \mathrm{m})$ for the TIMOT; these are placed in water cooled buckets outside the vacuum and are brought close to the trapping region by placing them inside reentrant glass windows situated on either side of the chamber.

Both helium isotopes are collimated, slowed, and confined in the TIMOT using $1083 \mathrm{~nm}$ light nearly resonant with their $2{ }^{3} S_{1} \rightarrow 2{ }^{3} P_{2}$ optical transitions (see Fig. 5 ) (natural linewidth $\Gamma / 2 \pi=1.62 \mathrm{MHz}$ and saturation intensity $I_{\text {sat }}=0.166 \mathrm{~mW} \mathrm{~cm}^{-2}$, for the cycling transition). As the isotope shift for this transition is $\approx 34 \mathrm{GHz}$ the bichromatic beams are produced by overlapping the output from two ytterbium-doped fiber lasers (IPG Photonics) on a non-polarizing 50/50 beam splitter. One beam is sent to the collimation section, whilst the second is coupled into a single mode polarization maintaining fiber to ensure a perfect overlap of the two frequency components, before being split into the Zeeman slowing beam and the trapping beams. Each fiber laser is locked to the respective cooling transition using saturated absorption spectroscopy in an rf-discharge cell; acousto-optic modulators are then used to generate the slowing and trapping frequencies which are detuned by $-500 \mathrm{MHz}$ and $-40 \mathrm{MHz}$ respectively (see Fig. 51). The slowing beam is focused on the source and has a $1 / e^{2}$ intensity width of $2.2 \mathrm{~cm}$ 

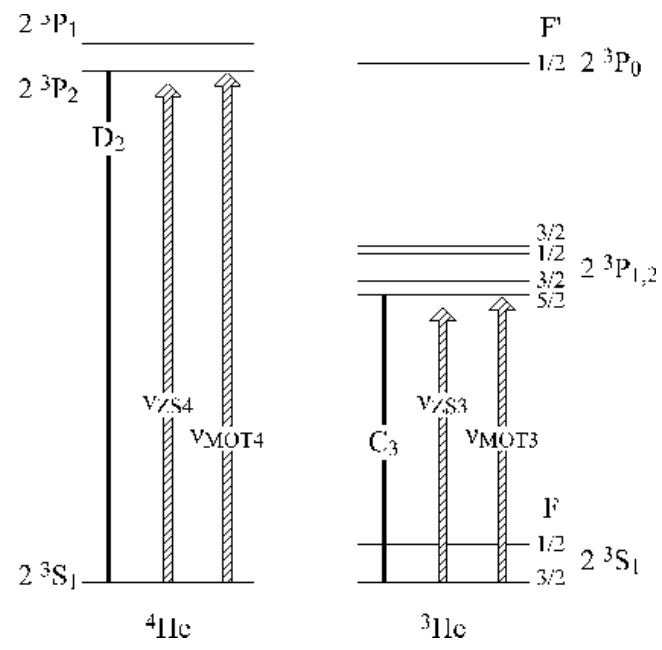

FIG. 5: Level scheme for the ground and first excited states in ${ }^{4} \mathrm{He}^{*}$ and ${ }^{3} \mathrm{He}^{*}$.

at the position of the trapped cloud, with each frequency component having a peak intensity of $I_{\text {peak }}=9 \mathrm{~mW} \mathrm{~cm}^{-2}$ $\left(I_{\text {peak }} / I_{\text {sat }} \approx 54\right)$, while the trapping beam is split into six independent Gaussian beams with $1 / e^{2}$ intensity widths of $1.8 \mathrm{~cm}$ and total peak intensity $I_{\text {peak }}=57 \mathrm{~mW} \mathrm{~cm}^{-2}$ $\left(I_{\text {peak }} / I_{\text {sat }} \approx 335\right)$. The outputs of two diode lasers (linewidths $<500 \mathrm{kHz}$ ), each locked using saturated absorption spectroscopy to the helium $2{ }^{3} S_{1} \rightarrow 2{ }^{3} P_{2}$ resonance of one of the isotopes, are overlapped on a second non-polarizing beam-splitter cube and coupled into a single mode polarization maintaining fiber. This system delivers two, weak, linearly polarized probe beams $(\Delta=0$, $I=0.05 I_{\text {sat }}$, with $I_{\text {sat }}=0.27 \mathrm{~mW} \mathrm{~cm}^{-2}$ assuming equal population of all magnetic sublevels of the $2{ }^{3} S_{1}$ state in the trap) to the chamber for the absorption imaging of each component of the trapped cloud. Absorption images are recorded using an IR-sensitive charge-coupled device (CCD) camera [37]. Unfortunately the imaging of both trapped components cannot be carried out in a single experimental cycle; sequential runs must be made, imaging first one component and then the other.

Trapped clouds are further monitored by two microchannel plate (MCP) detectors mounted inside the chamber. Operated at a voltage of $-1.5 \mathrm{kV}$ and positioned $11 \mathrm{~cm}$ from the center of the trap, the MCP's are used to independently monitor the ions and $\mathrm{He}^{*}$ atoms escaping or released from the trap. With an exposed front plate held at negative high voltage, one MCP mounted above the trap center attracts all positive ions produced during ionizing collisions (Eq. (1)) in the trap. The second MCP is shielded by a grounded grid, mounted below the trap center, and detects only $\mathrm{He}^{*}$ atoms.

The shielded MCP is used to perform time-of-flight (TOF) measurements from which we determine the temperature of the trapped atoms. An absorpton image, in combination with the measured temperature, then allows us to determine the density distribution, size, and

TABLE III: Typical experimental values of the ${ }^{3} \mathrm{He}^{*}$ and ${ }^{4} \mathrm{He}^{*}$ components in our TIMOT (error bars correspond to 1 standard deviation).

\begin{tabular}{lcc}
\hline \hline & ${ }^{3} \mathrm{He}^{*}$ & ${ }^{4} \mathrm{He}^{*}$ \\
\hline Temperature $T(\mathrm{mK})$ & $1.2(1)$ & $1.2(1)$ \\
Number of atoms $N$ & $1.0(3) \times 10^{8}$ & $1.3(3) \times 10^{8}$ \\
Central density $n_{0}\left(\mathrm{~cm}^{-3}\right)$ & $0.5(1) \times 10^{9}$ & $1.0(2) \times 10^{9}$ \\
Axial radius $\sigma_{p}(\mathrm{~cm})$ & $0.28(4)$ & $0.25(3)$ \\
Radial radius $\sigma_{z}(\mathrm{~cm})$ & $0.16(2)$ & $0.14(1)$ \\
\hline \hline
\end{tabular}

absolute atom number of the sample. We then use the unshielded MCP to measure the instantaneous ionization rate in the trapped sample, which, in combination with the information obtained from the absorption images, allows us to determine trap loss and ionization rates in the sample. Our typical TIMOT parameters and measurements have been reported previously [13, 19] and are given in Table III. The lower temperatures realized in the present experiments are due to the resolution of a power imbalance in two of the trapping laser beams.

\section{DETERMINATION OF THE HETERONUCLEAR LOSS RATE}

The time evolution of the total number of atoms trapped in our TIMOT, $N=N_{3}+N_{4}$, may be described by the following phenomenological equation [2]:

$$
\frac{d N}{d t}=L_{3}-\alpha_{3} N_{3}(t)-\beta_{33} \iiint n_{3}^{2}(\mathbf{r}, t) d^{3} \mathbf{r}+L_{4}-\alpha_{4} N_{4}(t)-\beta_{44} \iiint n_{4}^{2}(\mathbf{r}, t) d^{3} \mathbf{r}-\beta_{34} \iiint n_{3}(\mathbf{r}, t) n_{4}(\mathbf{r}, t) d^{3} \mathbf{r},
$$

where $t$ denotes time, $L$ is the rate at which atoms are loaded into the TIMOT, $\alpha$ is the linear loss rate coef- ficient describing collisions between trapped $\mathrm{He}^{*}$ atoms and background gases, $\beta$ is the loss rate coefficient result- 
ing from binary collisions between trapped $\mathrm{He}^{*}$ atoms, and $n$ is the cloud density. Subscripts denote whether a given parameter pertains to either of the ${ }^{3} \mathrm{He}^{*}$ or ${ }^{4} \mathrm{He}^{*}$ components of the mixture.

It should be noted at this point that the "density limited" regime, often mentioned with regard to the alkali systems, is not a feature of the metastable noble gas systems; in the latter the density is not limited by radiation trapping within the cloud, but by ionizing colli- sional losses. This is born out in Eq. (12) by the time dependance of the atom density distributions and hence our inability to make the simplifying constant density approximation in the following experiments.

Both linear and quadratic losses in Eq. (12) are due to a number of different mechanisms, and may be subdivided into either ionizing or non-ionizing categories. The ion production rate may then be expressed in a manner analogous to Eq. (12):

$$
\frac{d N_{\text {ion }}}{d t}=\epsilon_{a} \alpha_{3} N_{3}(t)+\epsilon_{b} K_{33} \iiint n_{3}^{2}(\mathbf{r}, t) d^{3} \mathbf{r}+\epsilon_{c} \alpha_{4} N_{4}(t)+\epsilon_{d} K_{44} \iiint n_{4}^{2}(\mathbf{r}, t) d^{3} \mathbf{r}+\epsilon_{e} K_{34} \iiint n_{3}(\mathbf{r}, t) n_{4}(\mathbf{r}, t) d^{3} \mathbf{r},
$$

where $\epsilon_{a}, \epsilon_{b}, \epsilon_{c}, \epsilon_{d}$ and $\epsilon_{e}$ are the weights of the various ionization mechanisms (and may include a factor to account for a less than unity detection efficiency), and the collision rate coefficient $K$ has been introduced. The loss and collision rate coefficients are related by the equation $\beta=2 K$, and the appearance of $K$, instead of $\beta$ in Eq. 13 expresses the fact that during each ionizing collision, one ion is produced, but two atoms are lost from the trap.
From an analysis of the trap loss mechanisms [13, 24] it can be seen that to a good approximation $\epsilon_{a}=\epsilon_{c}=0$, whilst $\epsilon_{b}=\epsilon_{d}=\epsilon_{e}$. The current signal measured by the $\mathrm{MCP}$ is proportional to the ionization rate, Eq. (13); and for gaussian spatial density distributions (centered with respect to each other), the voltage measured by the oscilloscope may then be written as

$$
\phi_{\text {TIMOT }}(t)=e R_{\mathrm{eff}}\left[K_{33} n_{03}^{2}(t)\left(\pi \sigma_{3}^{2}\right)^{\frac{3}{2}}+K_{44} n_{04}^{2}(t)\left(\pi \sigma_{4}^{2}\right)^{\frac{3}{2}}+K_{34} n_{03}(t) n_{04}(t)\left[\frac{2 \pi \sigma_{3}^{2} \sigma_{4}^{2}}{\sigma_{3}^{2}+\sigma_{4}^{2}}\right]^{\frac{3}{2}}\right]+\phi_{\mathrm{bgr}}
$$

where $n_{0}$ is the central density, $\sigma$ is the mean rms radius (of a given cloud component), $e$ is the electron charge, and $R_{\text {eff }}$ is an effective resistance.

The experiment is based around the ability of an MCP detector to measure the ions produced in our metastable isotopic mixture with very high efficiency, and employs a method first used by Bardou et al. [5] to determine the ionization rate in the absence of light. To measure the rate in the dark we perform an experiment in which we load the TIMOT, switch off the Zeeman slower beam and all MOT beams using the frequency detuning AOM's (the quadrupole field remains on) for $100 \mu \mathrm{s}$, before switching the slowing and trapping light back on again. This on/off cycle is easily repeated many times while we monitor the ion signal and average it (see Fig. 6). The switch-off time is short compared to the dynamics of the expanding cloud (we see no variation in the ion signal during the switch off period) and we switch the light on long enough (200 ms) to recapture the cloud and allow it to equilibrate.

Equation (14) describes the ion production rate in the dark of our TIMOT; a similar equation describes the ion production rate of a single-isotope MOT, $\phi_{M O T}$. By combining the equations for $\phi_{T I M O T}$ and $\phi_{M O T}$ with a measurement of the ratio $r=\left(\phi_{T I M O T}-\phi_{b g r}\right) /\left(\phi_{M O T}-\right.$ $\left.\phi_{\text {bgr }}\right)$ (where the time dependance of the measured signals has been omitted because of the short duration of the switch-off period), we can derive an expression for $K_{34}$. It has been verified that under our experimental conditions the MCP signal varies linearly with the ion production rate, and all cloud densities and radii may be derived from absorption images, while we have previously measured $K_{33}$ and $K_{44}[13]$. As the trap parameters, and therefore the distribution over the magnetic substates of the atoms, have changed since the experiments described in Ref. 13] were performed, we have used the theory described in Sec. IIB to correct the measured values of $K_{33}$ and $K_{44}$ for these effects, yielding: $K_{33}=$ $1.6(3) \times 10^{-10} \mathrm{~cm}^{3} / \mathrm{s}$ and $K_{44}=6.5(2) \times 10^{-11} \mathrm{~cm}^{3} / \mathrm{s}$.

\section{RESULTS AND DISCUSSION}

Performing the experiments described in the previous section, we obtain the result: $K_{34}^{(e x p)}=2.5(8) \times$ $10^{-10} \mathrm{~cm}^{3} / \mathrm{s}$, which compares well with the theoretical prediction, $K_{34}^{(\mathrm{th})}=2.4 \times 10^{-10} \mathrm{~cm}^{3} / \mathrm{s}$, obtained after 


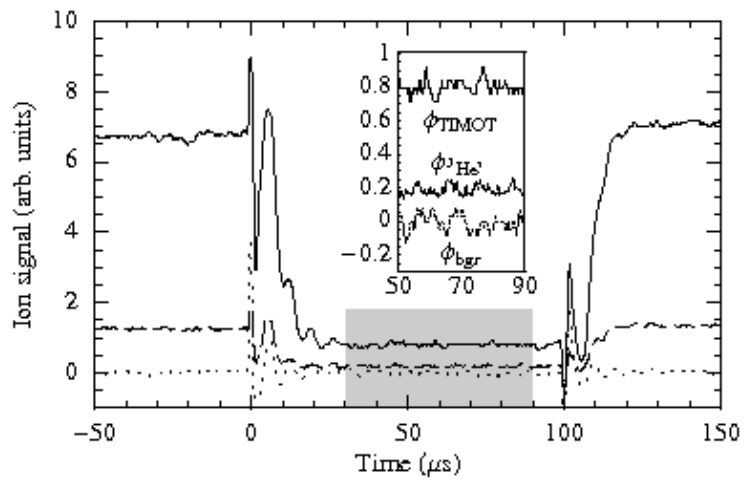

FIG. 6: Averaged ion signals measured during the experiments. The full line is the signal recorded whilst performing the experiment with a TIMOT, the dashed line is the corresponding curve for a ${ }^{3} \mathrm{He}^{*} \mathrm{MOT}$ (the analogous signal for a ${ }^{4} \mathrm{He}^{*}$ MOT can also be obtained), and the dotted line is the background signal. The ion rate measured is then averaged over the $60 \mu s$ interval indicated by the shaded region in order to obtain $\left(\phi_{\text {TIMOT }}-\phi_{\text {bgr }}\right)$ and $\left(\phi_{3 \mathrm{He}^{*} /{ }^{4} \mathrm{He}^{*}}-\phi_{\mathrm{bgr}}\right)$.

undertaking the calculation described in Sec. II For future comparison with other theoretical models, the equivalent theoretical loss-rate coefficient for an unpolarized gas mixture at a temperature of $T=1 \mathrm{mK}$ is $K_{34}^{(\text {unpol) }}=2.9 \times 10^{-10} \mathrm{~cm}^{3} / \mathrm{s}$ (see Fig. 3). These numbers describe the total heteronuclear loss rate in the absence of light; the only other potential loss process would be hyperfine state changing collisions, however, due to the inverted nature of the hyperfine structure in ${ }^{3} \mathrm{He}^{*}$ the atoms occupy the lowest hyperfine level of the $2{ }^{3} S_{1}$ multiplet and so cannot relax to a lower level, while the endothermic collision necessary to reach the $\mathrm{F}=1 / 2$ state would require $\approx 200 \mathrm{mK}$ to be provided by the trap, 200 times more than the typical collision energy in the TIMOT. The error in the experimental value is mainly determined by errors in the absorption imaging, which we find to be $\approx 30 \%$, and by the error bars reported on our previous measurements of the homonuclear loss rates [13].

With regard to optically assisted collisions, we note that the excited state potentials in the case of heteronuclear collisions are governed at long-range by the van-derWaals interaction $\left(\propto 1 / \mathrm{R}^{6}\right)$, having a much shorter range than that of the resonant dipole interaction $\left(\propto 1 / R^{3}\right)$ dominant in the homonuclear case. In our TIMOT the laser beams are, in contrast to most experiments performed on heteronuclear collisions, far-detuned (25 natural linewidths) from resonance and the atomic excited state population in the trap is therefore negligible. We can only excite a molecular state if the correct light frequency is present and the atoms have reached the Condon point for the transition. As all light frequencies in our TIMOT are far detuned from any heteronuclear transition we expect no contribution to the trap loss rate from optically assisted heteronuclear collisions.

To summarize, we have measured the heteronuclear loss rate coefficient $K_{34}^{(e x p)}$ in the absence of light for a trapped mixture of ${ }^{3} \mathrm{He}^{*}$ and ${ }^{4} \mathrm{He}^{*}$ atoms at $T=1 \mathrm{mK}$. The measured value of $K_{34}^{(e x p)}$ compares very well with the value predicted by our single channel model of ionizing collisions in the He* system. Recently, both helium isotopes were magnetically trapped in the multi-partial wave regime using buffer-gas cooling, and a deep magnetic trap [38]. The probable observation of Penning ionization under these conditions has been reported [39], and it would be interesting to extend our theory into the multi-partial wave regime and to high magnetic field values. With the production of quantum degenerate mixtures of ${ }^{3} \mathrm{He}^{*}$ and ${ }^{4} \mathrm{He}^{*}$ [40], we also have the possibility of investigating both homonuclear and heteronuclear $\mathrm{He}^{*}$ collisions at ultracold temperatures in greater detail. An experiment of interest in this area would be the implementation of an optical dipole trap in which it would be possible to prepare ultracold samples in well defined magnetic substates. In particular, it would be possible to prepare trapped ultracold samples of ${ }^{3} \mathrm{He}^{*}\left({ }^{4} \mathrm{He}^{*}\right)$ in the $m_{F}=-3 / 2\left(m_{J}=-1\right)$ states, for which (as in the magnetically trapped $m_{F}=+3 / 2\left(m_{J}=+1\right)$ states used in the production of ultracold He* gases) Penning ionization should be suppressed.

\section{Acknowledgments}

We thank Jacques Bouma for technical support. This work was supported by the "Cold Atoms" program of the Dutch Foundation for Fundamental Research on Matter (FOM), the Space Research Organization Netherlands (SRON), Grant No. MG-051, and the European Union, Grant No. HPRN-CT-2000-00125.
[1] J. Weiner, Cold and ultracold collisions in quantum microscopic and mesoscopic systems (Cambridge University Press, 2003).

[2] M. Prentiss, A. Cable, J. E. Bjorkholm, S. Chu, E. I. Raab, and D. E. Pritchard, Opt. Lett. 13, 452 (1988).

[3] D. Sesko, T. Walker, C. Monroe, A. Gallagher, and C. Wieman, Phys. Rev. Lett. 63, 961 (1989).
[4] C. D. Wallace, T. P. Dinneen, Kit-Yan N. Tan, T. T. Grove, and P. L. Gould, Phys. Rev. Lett. 69, 897 (1992).

[5] F. Bardou, O. Emile, J. M. Courty, C. I. Westbrook, and A. Aspect, Europhys. Lett. 20, 681 (1992).

[6] L. Marcassa, V. Bagnato, Y. Wang, C. Tsao, J. Weiner, O. Dulieu, Y. B. Band, and P. S. Julienne, Phys. Rev. A 47, R4563 (1993). 
[7] J. Kawanaka, K. Shimizu, H. Takuma, and F. Shimizu, Phys. Rev. A 48, R883 (1993).

[8] H. Katori and F. Shimizu, Phys. Rev. Lett. 73, 2555 (1994).

[9] M. Walhout, U. Sterr, C. Orzel, M. Hoogerland, and S. L. Rolston, Phys. Rev. Lett. 74, 506 (1995).

[10] N. W. M. Ritchie, E. R. I. Abraham, Y. Y. Xiao, C. C. Bradley, R. G. Hulet, and P. S. Julienne, Phys. Rev. A 51, R890 (1995).

[11] R. S. Williamson III and T. Walker, J. Opt. Soc. Am. B 12, 1393 (1995).

[12] H. Katori, H. Kunugita, and T. Ido, Phys. Rev. A 52, R4324 (1995).

[13] R. J. W. Stas, J. M. McNamara, W. Hogervorst, and W. Vassen, Phys. Rev. A 73, 032713 (2006).

[14] W. Süptitz, G. Wokurka, F. Strauch, P. Kohns, and W. Ertmer, Opt. Lett. 19, 1571 (1994).

[15] M. Prevedelli, F. S. Cataliotti, E. A. Cornell, J. R. Ensher, C. Fort, L. Ricci, G. M. Tino, and M. Inguscio, Phys. Rev. A 59, 886 (1999).

[16] M.-O. Mewes, G. Ferrari, F. Schreck, A. Sinatra, and C. Salomon, Phys. Rev. A 61, 011403(R) (1999).

[17] S. G. Crane, X. Zhao, W. Taylor, and D. J. Vieira, Phys. Rev. A 62, 011402(R) (2000).

[18] T. Loftus, J. R. Bochinski, and T. W. Mossberg, Phys. Rev. A 63, 053401 (2001).

[19] R. J. W. Stas, J. M. McNamara, W. Hogervorst, and W. Vassen, Phys. Rev. Lett. 93, 053001 (2004).

[20] M. Kumakura and N. Morita, Phys. Rev. Lett. 82, 2848 (1999).

[21] H. C. Mastwijk, J. W. Thomsen, P. van der Straten, and A. Niehaus, Phys. Rev. Lett. 80, 5516 (1998).

[22] P. J. J. Tol, N. Herschbach, E. A. Hessels, W. Hogervorst, and W. Vassen, Phys. Rev. A 60, R761 (1999).

[23] N. Herschbach, P. J. J. Tol, W. Hogervorst, and W. Vassen, Phys. Rev. A 61, 050702(R) (2000).

[24] R. J. W. Stas, Ph.D thesis, Vrije Universiteit, Amsterdam (2005).
[25] V. Venturi, I. B. Whittingham, P. J. Leo and G. Peach, Phys. Rev. A 60, 4635 (1999).

[26] V. Venturi and I. B. Whittingham, Phys. Rev. A 61, 060703(R) (2000).

[27] P. J. Leo and V. Venturi and I. B. Whittingham and J. F. Babb, Phys. Rev. A 64, 042710 (2001).

[28] A. J. Yencha, in Electron Spectroscopy: Theory, techniques and Applications, edited by C. R. Brundle and A. D. Baker (Academic Press, London, 1984), Vol. 5, pp. 197-373.

[29] H. S. W. Massey, Electronic and ionic impact phenomena, volume 3 (Oxford University Press, 1971).

[30] P. O. Fedichev, M. W. Reynolds, U. M. Rahmanov, and G. V. Shlyapnikov, Phys. Rev. A 53, 1447 (1996).

[31] M. W. Müller, A. Merz, M.-W. Ruf, H. Hotop, W. Meyer, and M. Movre, Z. Phys. D 21,89 (1991).

[32] C. Orzel, M. Walhout, U. Sterr, P. S. Julienne, and S. L. Rolston, Phys. Rev. A 59, 1926 (1999).

[33] P. S. Julienne and F. H. Mies, J. Opt. Soc. Am. B 6, 2257 (1989).

[34] C. Cohen-Tannoudji, B. Diu, and F. Laloë, Quantum Mechanics (John Wiley \& Sons, New York, 1977).

[35] W. Rooijakkers, W. Hogervorst, and W. Vassen, Opt. Commun. 123, 321 (1996).

[36] A. S. Tychkov, T. Jeltes, J. M. McNamara, P. J. J. Tol, N. Herschbach, W. Hogervorst, and W. Vassen, Phys. Rev. A 73, 031603(R) (2006).

[37] P. J. J. Tol, Ph.D. thesis, Vrije Universiteit, Amsterdam (2005).

[38] S. V. Nguyen, S. Charles Doret, C. B. Connolly, R. A. Michniak, W. Ketterle, and J. M. Doyle, Phys. Rev. A 72, 060703(R) (2005).

[39] S. Nguyen, Ph.D thesis, Harvard University, Cambridge, Massachusetts (2006).

[40] J. M. McNamara, T. Jeltes, A. S. Tychkov, W. Hogervorst and W. Vassen, Phys. Rev. Lett. 97, 080404 (2006). 